\title{
Mechanism of action of EGFR mutations
}

The role of mutant epidermal growth factor receptor (EGFR) kinases in the growth of a host of human cancers including lung cancer is well known. The activation of wild type EGFR is primarily driven by the binding of growth factors leading to receptor dimerisation.

In this paper the authors, through a series of simulation experiments, show that the wildtype EGFR kinase domain is intrinsically disordered and it becomes ordered (active) only upon dimerisation. This intrinsic disorder (partial unfolding) is a third functionally important conformation in addition to the previously identified ' $\alpha \mathrm{C}$-out inactive' and ' $\alpha \mathrm{C}$-in active' conformations. EGFR signalling involves multiple layers of controlling mechanisms; the identification of an intrinsically disordered state of EGFR kinase introduces another element critical for its function.

EGFR mutations were generally assumed to give rise to spontaneous kinase activation, eliminating the dependence of the cancer mutants on dimerisation for activation. However the authors have been able to show that higher kinase activity of EGFR mutants like L834R mutation is due to a decreased threshold for dimerisation mediated activation (by suppression of the local intrinsic disorder) rather than to enhanced intrinsic catalytic potency of the mutant. The authors also found that phosphorylation of EGFR kinase domain at TYR 845 may suppress the intrinsic disorder and promote its dimerisation. This could potentially be a molecular mechanism for autonomous EGFR signalling.

- Shan Y, Eastwood MP, Zhang X, et al. Oncogenic mutations counteract intrinsic disorder in the EGFR kinase and promote receptor dimerization. Cell 2012;149:860-70.

Raja Reddy

Correspondence to Dr Raja Reddy, Department of Respiratory Medicine, Kettering Hospital, Kettering, UK; rvreddy51@hotmail.com

To cite Reddy R. Thorax 2013;68:759.

Published Online First 2 October 2012

Thorax 2013;68:759. doi:10.1136/thoraxjnl-2012-202641 